\title{
Determinants of some special matrices over commutative finite chain rings
}

https://doi.org/10.1515/spma-2020-0118

Received August 11, 2020; accepted November 11, 2020

\begin{abstract}
Circulant matrices over finite fields and over commutative finite chain rings have been of interest due to their nice algebraic structures and wide applications. In many cases, such matrices over rings have a closed connection with diagonal matrices over their extension rings. In this paper, the determinants of diagonal and circulant matrices over commutative finite chain rings $R$ with residue field $\mathbb{F}_{q}$ are studied. The number of $n \times n$ diagonal matrices over $R$ of determinant $a$ is determined for all elements $a$ in $R$ and for all positive integers $n$. Subsequently, the enumeration of nonsingular $n \times n$ circulant matrices over $R$ of determinant $a$ is given for all units $a$ in $R$ and all positive integers $n$ such that $\operatorname{gcd}(n, q)=1$. In some cases, the number of singular $n \times n$ circulant matrices over $R$ with a fixed determinant is determined through the link between the rings of circulant matrices and diagonal matrices. As applications, a brief discussion on the determinants of diagonal and circulant matrices over commutative finite principal ideal rings is given. Finally, some open problems and conjectures are posted
\end{abstract}

Keywords: Determinants, Diagonal matrices, Circulant matrices, Commutative finite chain rings

MSC: 15A15, 11C20, 15B33, 13F10

\section{Introduction}

Circulant matrices have been introduced in [3] and extensively been studied due to their nice algebraic structures, wide applications, various links with other objects. The book "Circulant Matrices" [6] has summarized algebraic structures, properties and applications of such matrices. Later, circulant matrices have been shown to have applications in many disciplines, e.g., signal processing, image processing, networked systems, communications, and coding theory. Especially, (nonsingular) circulant matrices over finite fields and over commutative finite chain rings are applied in constructions of various families of linear codes (see [1], [2], [6], [9], [10], [11], [14], [17], [18], [19], and references therein). Circulant matrices have shown to have a closed connection with diagonal matrices (see, for example, [6] and [14]). Therefore, some properties of circulant matrices can be determined in terms of diagonal matrices.

Determinants of matrices are known for their useful properties and applications in linear algebra, matrix theory, and other braces of Mathematics and Engineering. Since the singularity of matrices is useful in applications, determinants and related properties of matrices have been extensively studied. The number of $n \times n$ singular (resp., nonsingular) matrices over a finite field has been given in [15]. The number of $n \times n$ matrices over commutative finite chain rings (CFCRs) of a fixed determinant has been completely determined in [5]. Diagonal and circulant matrices are two interesting subfamilies of the ones in [5]. Therefore, it is of natural interest to study the determinants of such matrices over CFCRs.

The paper is organized as follows. Some definitions and useful properties of CFCRs $R$ and matrices are recalled in Section 2. In Section 3, the number $d_{n}(R, a)$ of $n \times n$ diagonal matrices over $R$ of determinant $a$ is

^Corresponding Author: Somphong Jitman: Department of Mathematics, Faculty of Science, Silpakorn University, Nakhon Pathom 73000, Thailand, E-mail:sjitman@gmail.com 
established for all elements $a \in R$ and for all positive integers $n$. In Section 4, the number $c_{n}(R, a)$ of $n \times n$ circulant matrices over $R$ is studied. In the case where $a$ is a unit in $R, c_{n}(R, a)$ is determined for all positive integers $n$ such that $\operatorname{gcd}(n, q)=1$. For a non-unit $a \in R$, the number $c_{n}(R, a)$ is given for all positive integers $n$ such that $n \mid(q-1)$. Summary, remarks, conjectures, and open problems are given in Section 5.

\section{Preliminaries}

Definitions, notations, and some useful properties of commutative finite chain rings and matrices are recalled.

\subsection{Commutative Finite Chain Rings}

A ring $R$ with identity $1 \neq 0$ is called a commutative finite chain ring (CFCR) if it is finite, commutative, and its ideals are linearly ordered by inclusion. The properties of CFCRs required in this paper are recalled in the discussion below. The reader is referred to [8], [12], and [13] for more details on CFCRs.

A CFCR is known to be a principal ideal ring and its maximal ideal is unique. Let $R$ be a CFCR whose maximal ideal is generated by $\gamma$. The ideals in $R$ can be written in the forms of

$$
R \supsetneq \gamma R \supsetneq \gamma^{2} R \supsetneq \cdots \supsetneq \gamma^{e-1} R \supsetneq \gamma^{e} R=\{0\},
$$

for some positive integer $e$. The nilpotency index of a CFCR $R$ is defined to be the smallest positive integer $e$ such that $\gamma^{e}=0$. The quotient ring $R / \gamma R$ forms a finite field referred as the residue field of $R$. The cardinality and the characteristic of $R$ are powers of the characteristic of $R / \gamma R$. For a CFCR $R$, let $U(R)$ denote the set of units in $R$ and let $Z(R)$ denote the set of zero-divisors in $R$. The properties of a CFCR in the next lemma are well known.

Lemma 2.1 ([12] and [13]). Let $R$ be a CFCR of nilpotency index $e$ and let $\gamma$ be a generator of the maximal ideal of $R$. Let $V \subseteq R$ be a set of representatives for the equivalence classes of $R$ under congruence modulo $\gamma$. Assume that the residue field $R /\langle\gamma\rangle \cong \mathbb{F}_{q}$ for some prime power $q$. Then the following statements hold.

1) For each $r \in R$, there exist unique $a_{0}, a_{1}, \ldots a_{e-1} \in V$ such that

$$
r=a_{0}+a_{1} \gamma+\cdots+a_{e-1} \gamma^{e-1} .
$$

2) $|V|=q$.

3) $\left|\gamma^{j} R\right|=q^{e-j}$ for all $0 \leq j \leq e$.

4) $U(R)=\{a+\gamma b \mid a \in V \backslash\{0\}$ and $b \in R\}$.

5) $|U(R)|=(q-1) q^{e-1}$.

6) For each $0 \leq i \leq e, R / \gamma^{i} R$ is a CFCR of nilpotency index $i$ and residue field $\mathbb{F}_{q}$.

From Lemma 2.1, it can be deduced that $Z(R)=\gamma R \backslash\{0\},|Z(R)|=q^{e-1}-1$, and $R=\{0\} \cup Z(R) \cup U(R)$ is a disjoint union.

\subsection{Diagonal and Circulant Matrices}

Given a positive integer $n$ and a commutative ring $\mathfrak{R}$, an $n \times n$ matrix $A$ over $\mathfrak{R}$ is called a diagonal matrix if $a_{i j}=0$ for all $i \neq j$. Denote by $\operatorname{diag}\left(a_{11}, a_{22}, \ldots, a_{n n}\right)$ the diagonal matrix $A$. It is well known that

$$
\operatorname{det}\left(\operatorname{diag}\left(a_{1}, a_{2}, \ldots, a_{n}\right)\right)=\prod_{i=1}^{n} a_{i} .
$$


Let $D_{n}(\mathfrak{R})=\left\{\operatorname{diag}\left(a_{1}, a_{2}, \ldots, a_{n}\right) \mid a_{i} \in \mathfrak{R}\right\}$ denote the set of $n \times n$ diagonal matrices over $\mathfrak{R}$. For each $n \in \mathbb{N}$ and $a \in \mathfrak{R}$, let $D_{n}(\Re, a)=\left\{A \in D_{n}(\Re) \mid \operatorname{det}(A)=a\right\}$ and $d_{n}(\mathfrak{R}, a)=\left|D_{n}(\mathfrak{R}, a)\right|$.

An $n \times n$ matrix $A$ over $\mathfrak{R}$ is called a circulant matrix if $A$ is of the form

$$
A=\left[\begin{array}{ccccc}
a_{1} & a_{2} & a_{3} & \ldots & a_{n} \\
a_{n} & a_{1} & a_{2} & \ldots & a_{n-1} \\
a_{n-1} & a_{n} & a_{1} & \ldots & a_{n-2} \\
\vdots & \vdots & \vdots & \ddots & \vdots \\
a_{2} & a_{3} & a_{4} & \ldots & a_{1}
\end{array}\right],
$$

for some $a_{1}, a_{2}, \ldots, a_{n}$ in $\mathfrak{R}$, and denote it by $\operatorname{cir}\left(a_{1}, a_{2}, \ldots, a_{n}\right)$. If there exists an extension ring of $\Re$ containing a primitive $n$th root of unity, say $\omega$, the eigenvalues and determinant of a circulant matrix $A=\operatorname{cir}\left(a_{1}, a_{2}, \ldots, a_{n}\right)$ over $\mathfrak{R}$ can be given as follows. From [6] and [14], the eigenvalues of $A$ are of the form

$$
\omega_{j}=\sum_{i=1}^{n} a_{i} \omega^{(i-1) j}
$$

for all $0 \leq j \leq n-1$. It follows that

$$
\operatorname{det}(A)=\prod_{j=0}^{n} \omega_{j}=\prod_{j=0}^{n-1}\left(\sum_{i=1}^{n} a_{i} \omega^{(i-1) j}\right) .
$$

Let $C_{n}(\mathfrak{R})=\left\{\operatorname{cir}\left(a_{1}, a_{2}, \ldots, a_{n}\right) \mid a_{i} \in \mathfrak{R}\right\}$ denote the set of $n \times n$ circulant matrices over the ring $\mathfrak{R}$. Let $C_{n}(\Re, a)=\left\{A \in C_{n}(\Re) \mid \operatorname{det}(A)=a\right\}$ be the set of $n \times n$ circulant matrices over $\mathfrak{R}$ whose determinant is $a$ and let $c_{n}(\mathfrak{R}, a)=\left|C_{n}(\Re, a)\right|$.

In this paper, we focus on the numbers $c_{n}(R, a)$ and $d_{n}(R, a)$ in the case where $R$ is CFCRs and $a \in R$ which are established in Section 3 and Section 4, respectively. While some techniques for $n \times n$ matrices from [5] are applied, the detailed proofs and counting are slightly different. For completeness, the full proofs and counting are given. The reader may refer to [5] for comparison.

\section{Determinants of Diagonal Matrices over CFCRs}

Determinants of diagonal matrices over a CFCR $R$ are focused on and the number $d_{n}(R, a)$ is completely determined for all positive integers $n$ and for all $a \in R$.

For a CFCR $R$ of nilpotency index $e$ and residue field $\mathbb{F}_{q}$, let $\gamma$ be a generator of its maximal ideal. For each $a \in R$, it is easily seen that $a=\gamma^{s} b$ for some $0 \leq s \leq e$ and unit $b \in U(R)$ by Lemma 2.1. Precisely, $a$ is a unit if $s=0, a=\gamma^{s} b$ is a zero-divisor if $1 \leq s \leq e-1$, and $a=0$ if $s=e$.

For each $n \in \mathbb{N}$ and $a \in R$, the number $d_{n}(R, a)$ are determined in this section. The over view results are summarized in Figure 1 and the details are given right after.

First, we begin with a reduced formula for the number $d_{n}(R, a)$.

Theorem 3.1. Let $R$ be a CFCR of nilpotency index e and let $n$ be a positive integer. If the maximal ideal of $R$ is generated by $\gamma$ and $0 \leq s \leq e$, then the following statements hold.

1) $d_{n}(R, a)>0$ for all elements $a \in R$.

2) $d_{n}\left(R, \gamma^{S}\right)=d_{n}\left(R, b \gamma^{S}\right)$ for all units $b$ in $U(R)$.

Proof. Since $\operatorname{det}(\operatorname{diag}(a, 1,1, \ldots, 1))=a$ for all elements $a \in R$, it follows that $\operatorname{diag}(a, 1,1, \ldots, 1) \in$ $D_{n}(R, a)$ which implies that $d_{n}(R, a)>0$ for all $a \in R$. This proves 1$)$. 


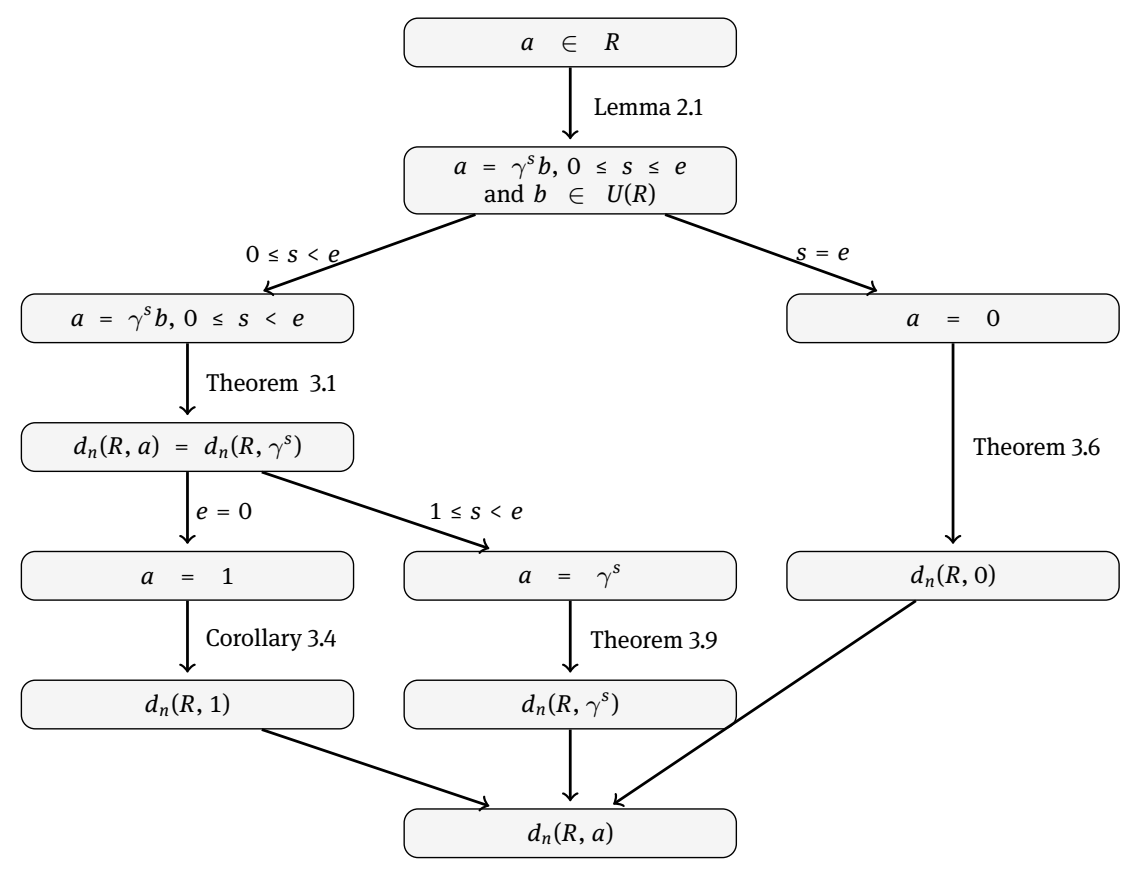

Figure 1: The number $d_{n}(R, a)$ over a CFCR $R$

To prove 2), let $b$ be a unit in $U(R)$ and let $0 \leq s \leq e$ be an integer. If $s=e$, then $\gamma^{s}=0=\gamma^{s} b$. Clearly, $d_{n}\left(R, \gamma^{S}\right)=d_{n}(R, 0)=d_{n}\left(R, b \gamma^{s}\right)$.

For each $0 \leq s<e$, let $\alpha: D_{n}\left(R, \gamma^{s}\right) \rightarrow D_{n}\left(R, b \gamma^{s}\right)$ be a map defined by

$$
\alpha(A)=\operatorname{diag}(b, 1,1, \ldots, 1) A
$$

for all $A \in D_{n}\left(R, \gamma^{S}\right)$. Since $\operatorname{diag}(b, 1,1, \ldots, 1)$ is nonsingular and $\operatorname{det}(A)=\gamma^{s}$ if and only if

$$
\operatorname{det}(\operatorname{diag}(b, 1,1, \ldots, 1) A)=b \operatorname{det}(A)=b \gamma^{s}
$$

for all $A \in D_{n}\left(R, \gamma^{s}\right), \alpha$ is a well-defined bijective map. Therefore,

$$
d_{n}\left(R, \gamma^{S}\right)=\left|D_{n}\left(R, \gamma^{S}\right)\right|=\left|D_{n}\left(R, b \gamma^{s}\right)\right|=d_{n}\left(R, b \gamma^{s}\right) .
$$

The second statement is proved.

By setting $s=0$, the next corollary follows.

Corollary 3.2. Let $R$ be a CFCR and let $n$ be a positive integer. Then $d_{n}(R, a)=d_{n}(R, 1)$ for all units $a \in U(R)$.

\subsection{Determinants of Nonsingular Diagonal Matrices over CFCRs}

In this subsection, nonsingular $n \times n$ diagonal matrices over CFCRs $R$ are focused on and the number $d_{n}(R, a)$ is determined for all positive integers $n$ and for all units $a \in U(R)$.

Let $N S D_{n}(R)=\left\{A \in D_{n}(R) \mid \operatorname{det}(A) \in U(R)\right\}$ denote the set of nonsingular $n \times n$ diagonal matrices over CFCRs $R$. Clearly,

$$
N S D_{n}(R)=\bigcup_{a \in U(R)} D_{n}(R, a)
$$

where the union is disjoint.

First, we determined the number $\left|N S D_{n}(R)\right|$ of nonsingular $n \times n$ diagonal matrices over CFCRs $R$. 
Lemma 3.3. Let $R$ be a CFCR of nilpotency index e and residue field $\mathbb{F}_{q}$ and let $n$ be a positive integer. Then

$$
\left|N S D_{n}(R)\right|=(q-1)^{n} q^{(e-1) n} .
$$

Proof. Since $\operatorname{det}\left(\operatorname{diag}\left(a_{1}, a_{2}, \ldots, a_{n}\right)\right) \in U(R)$ if and only if $a_{i} \in U(R)$ for all $1 \leq i \leq n$, we have $\left|N S D_{n}(R)\right|=$ $|U(R)|^{n}=(q-1)^{n} q^{(e-1) n}$ by Lemma 2.1.

By Corollary 3.2, $d_{n}(R, a)=d_{n}(R, 1)$ for all units $a \in R$, and hence, $d_{n}(R, 1)=\left|N S D_{n}(R)\right| /|U(R)|$. By Lemma 2.1 and Lemma 3.3, the result follows.

Corollary 3.4. Let $R$ be a CFCR of nilpotency index e and residue field $\mathbb{F}_{q}$ and let $n$ be a positive integer. Then

$$
d_{n}(R, a)=d_{n}(R, 1)=(q-1)^{(n-1)} q^{(e-1)(n-1)}
$$

for all $a \in U(R)$.

By setting $e=1$ in Lemma 3.3 and Corollary 3.4, it follows that

$$
\left|N S D_{n}\left(\mathbb{F}_{q}\right)\right|=(q-1)^{n} \text { and } d_{n}\left(\mathbb{F}_{q}, 1\right)=(q-1)^{n-1} .
$$

\subsection{Determinants of Singular Diagonal Matrices over CFCRs}

Determinants of singular $n \times n$ diagonal matrices over CFCRs $R$ are studied. Precisely, $d_{n}(R, 0)$ and $d_{n}(R, a)$ are determined for all zero-divisors $a \in Z(R)$.

\subsubsection{Singular Diagonal Matrices over CFCRs with Zero Determinant}

The recursive relation in the following lemma is key in determining $d_{n}(R, 0)$ in Theorem 3.6.

Lemma 3.5. Let $R$ be a CFCR of nilpotency index e and residue field $\mathbb{F}_{q}$ and let $\gamma$ be a generator of the maximal ideal of $R$. Then

$$
d_{n}(R, 0)=(q-1) q^{e-1} d_{n-1}(R, 0)+q^{n-1} d_{n}\left(R / \gamma^{e-1} R, 0+\gamma^{e-1} R\right)
$$

for all integers $n \geq 2$.

Proof. Let

$$
D_{n}^{\prime}(R, 0)=\left\{\operatorname{diag}\left(a_{1}, a_{2}, \ldots, a_{n}\right) \in D_{n}(R, 0) \mid a_{1} \in U(R)\right\}
$$

and

$$
D_{n}^{\prime \prime}(R, 0)=\left\{\operatorname{diag}\left(a_{1}, a_{2}, \ldots, a_{n}\right) \in D_{n}(R, 0) \mid a_{1} \notin U(R)\right\} .
$$

Let $v: D_{n}^{\prime}(R, 0) \rightarrow D_{n-1}(R, 0)$ be the map defined by

$$
\operatorname{diag}\left(a_{1}, a_{2}, \ldots, a_{n}\right) \mapsto \operatorname{diag}\left(a_{2}, \ldots, a_{n}\right) .
$$

It is not difficult to see that $v$ is a $(q-1) q^{e-1}$-to-one surjective map. For each $A \in D_{n}^{\prime}(R, 0)$, it can be seen that $\operatorname{det}(A)=0$ if and only if $\operatorname{det}(v(A))=0$. Consequently,

$$
\left|D_{n}^{\prime}(R, 0)\right|=(q-1) q^{e-1} d_{n-1}(R, 0) .
$$

For each $\operatorname{diag}\left(a_{1}, a_{2}, \ldots, a_{n}\right) \in D_{n}^{\prime \prime}(R, 0)$, we have $a_{1} \in \gamma R$ which implies that $a_{1}=\gamma b_{1}$ for some $b_{1} \in$ $\sum_{j=0}^{e-2} \gamma^{j} V$, where $V$ is given in Lemma 2.1. Let $\psi: D_{n}^{\prime \prime}(R, 0) \rightarrow D_{n}(R)$ be an injective map defined by

$$
\operatorname{diag}\left(a_{1}, a_{2}, \ldots, a_{n}\right) \mapsto \operatorname{diag}\left(b_{1}, a_{2}, \ldots, a_{n}\right) .
$$


Let $\beta: D_{n}(R) \rightarrow D_{n}\left(R / \gamma^{e-1} R\right)$ be a surjective ring homomorphism given by

$$
\beta(B)=\bar{B},
$$

where $\overline{\left[b_{i j}\right]}:=\left[b_{i j}+\gamma^{e-1} R\right]$ for all $\left[b_{i j}\right] \in D_{n}(R)$. For each $A \in D_{n}^{\prime \prime}(R, 0)$, $\operatorname{det}(A)=\gamma \operatorname{det}(\psi(A))$ which implies that $\operatorname{det}(A)=0$ if and only if $\operatorname{det}(\psi(A)) \in \gamma^{e-1} R$. Consequently, we have $\operatorname{det}(\beta(\psi(A)))=\operatorname{det}(\psi(A))+$ $\gamma^{e-1} R=0+\gamma^{e-1} R$. Hence, $\beta \circ \psi$ is surjective such that $\beta\left(\psi\left(D_{n}^{\prime \prime}(R, 0)\right)\right)=D_{n}\left(R / \gamma^{e-1} R, 0+\gamma^{e-1} R\right)$. For each $D \in D_{n}\left(R / \gamma^{e-1} R, 0+\gamma^{e-1} R\right)$, there are exactly $q^{n-1}$ matrices in $\psi\left(D_{n}^{\prime \prime}(R, 0)\right)$ whose images under $\beta$ are $D$. Since $\psi$ is an injective map, we have

$$
\left|D_{n}^{\prime \prime}(R, 0)\right|=q^{n-1} d_{n}\left(R / \gamma^{e-1} R, 0+\gamma^{e-1} R\right) .
$$

Since $D_{n}(R, 0)=D_{n}^{\prime}(R, 0) \cup D_{n}^{\prime \prime}(R, 0)$ is a disjoint union, the desired result follows.

Using the above relation, the number $d_{n}(R, 0)$ can be given in the next theorem.

Theorem 3.6. Let $R$ be a CFCR of nilpotency index e and residue field $\mathbb{F}_{q}$ and let $n$ be a positive integer. Then

$$
d_{n}(R, 0)=q^{n e}-(q-1)^{n} q^{(e-1) n} \sum_{i=0}^{e-1}\left(\begin{array}{c}
n+i-1 \\
n-1
\end{array}\right) q^{-i} .
$$

Proof. We prove (3.2) by induction on the nilpotency index $e$ and the size $n$ of the matrices. For $e=1$, we have $R=\mathbb{F}_{q}$ and (3.2) holds by (3.1), i.e.,

$$
d_{n}(R, 0)=d_{n}\left(\mathbb{F}_{q}, 0\right)=q^{n}-\left|N S D_{n}\left(\mathbb{F}_{q}\right)\right|=q^{n}-(q-1)^{n} .
$$

If $n=1$, then $d_{n}(R, 0)=1$ which coincides with (3.2).

Assume that the formula (3.2) holds true for the pairs (nilpotency index, size) in $\{(e-1, n),(e, n-1)\}$ for some positive integers $n \geq 2$ and $e \geq 2$. For $n \times n$ diagonal matrices over a CFCR $R$ of nilpotency index $e$, we have

$$
\begin{aligned}
d_{n}(R, 0)= & (q-1) q^{e-1} d_{n-1}(R, 0)+q^{n-1} d_{n}\left(R / \gamma^{e-1} R, 0+\gamma^{e-1} R\right) \\
& \text { by Lemma 3.5, } \\
= & (q-1) q^{e-1}\left(q^{(n-1) e}-(q-1)^{n-1} q^{(e-1)(n-1)} \sum_{i=0}^{e-1}\left(\begin{array}{c}
n+i-2 \\
n-2
\end{array}\right) q^{-i}\right) \\
& +q^{n-1}\left(q^{n(e-1)}-(q-1)^{n} q^{(e-2) n} \sum_{i=0}^{e-2}\left(\begin{array}{c}
n+i-1 \\
n-1
\end{array}\right) q^{-i}\right) \\
& \text { by the induction hypothesis, } \\
= & (q-1) q^{n e-1}-(q-1)^{n} q^{(e-1) n} \sum_{i=0}^{e-1}\left(\begin{array}{c}
n+i-2 \\
n-2
\end{array}\right) q^{-i} \\
& +q^{n e-1}-(q-1)^{n} q^{(e-1) n-1} \sum_{i=0}^{e-2}\left(\begin{array}{c}
n+i-1 \\
n-1
\end{array}\right) q^{-i} \\
= & (q-1) q^{n e-1}-(q-1)^{n} q^{(e-1) n} \sum_{i=0}^{e-1}\left(\begin{array}{c}
n+i-2 \\
n-2
\end{array}\right) q^{-i} \\
& +q^{n e-1}-(q-1)^{n} q^{(e-1) n} \sum_{i=0}^{e-1}\left(\begin{array}{c}
n+i-2 \\
n-1
\end{array}\right) q^{-i} \\
= & q^{n e}-(q-1)^{n} q^{(e-1) n} \sum_{i=0}^{e-1}\left(\left(\begin{array}{c}
n+i-2 \\
n-2
\end{array}\right)+\left(\begin{array}{c}
n+i-2 \\
n-1
\end{array}\right)\right) q^{-i} \\
= & q^{n e}-(q-1)^{n} q^{(e-1) n} \sum_{i=0}^{e-1}\left(\begin{array}{c}
n+i-1 \\
n-1
\end{array}\right) q^{-i} .
\end{aligned}
$$

The result follows immediately. 


\subsubsection{Singular Diagonal Matrices over CFCRs with Non-Zero Determinant}

Here, we focus on singular diagonal matrices whose determinants are non-zero elements. Precisely, the number $d_{n}(R, a)$ is determined for all zero-divisors $a \in Z(R)$. Note that for each zero-divisor $a \in Z(R)$, it can be written as $a=\gamma^{s} b$ for some $1 \leq s<e$ and $b \in U(R)$. By Theorem 3.1, it is enough to determine only $d_{n}\left(R, \gamma^{s}\right)$ for all integers $1 \leq s<e$.

The following results are key tools in determining $d_{n}\left(R, \gamma^{s}\right)$ in Theorem 3.9.

Lemma 3.7. Let $R$ be a CFCR of nilpotency index $e \geq 3$ and residue field $\mathbb{F}_{q}$ and let $\gamma$ be a generator of the maximal ideal of $R$. Then

$$
d_{n}\left(R, \gamma^{s}\right)=q^{(n-1)} d_{n}\left(R / \gamma^{e-1} R, \gamma^{s}+\gamma^{e-1} R\right)
$$

for all $1 \leq s<e-1$ and for all integers $n \geq 2$.

Proof. Let $n \geq 2$ and $1 \leq s<e-1$ be integers and let $\beta: D_{n}(R) \rightarrow D_{n}\left(R / \gamma^{e-1} R\right)$ be a ring homomorphism defined in the proof of Lemma 3.5 by

$$
\beta(A)=\bar{A},
$$

where $\overline{\left[a_{i j}\right]}:=\left[a_{i j}+\gamma^{e-1} R\right]$ for all $\left[a_{i j}\right] \in D_{n}(R)$. For each $A \in D_{n}(R)$, we have $\operatorname{det}(\beta(A))=\gamma^{S}+\gamma^{e-1} R$ if and only if $\operatorname{det}(A)=\gamma^{s}+\gamma^{e-1} b$ for some $b \in V$, where $V$ is given in Lemma 2.1. Since $1 \leq e-s-1<e-1$, the element $1+\gamma^{e-s-1} b$ is a unit in $U(R)$. Consequently,

$$
\begin{aligned}
\mid\left\{A \in D_{n}(R) \mid\right. & \left.\operatorname{det}(A)=\gamma^{s}+\gamma^{e-1} b \text { for some } b \in V\right\} \mid \\
& =\mid\left\{A \in D_{n}(R) \mid \operatorname{det}(A)=\gamma^{s}\left(1+\gamma^{e-s-1} b\right) \text { for some } b \in V\right\} \mid \\
& =\left|\left\{A \in D_{n}(R) \mid \operatorname{det}(A)=\gamma^{s}\right\}\right| \\
& =d_{n}\left(R, \gamma^{s}\right) .
\end{aligned}
$$

Equivalently,

$$
\left|\left\{A \in D_{n}(R) \mid \operatorname{det}(\beta(A))=\gamma^{s}+\gamma^{e-1} R\right\}\right|=|V| d_{n}\left(R, \gamma^{s}\right)=q d_{n}\left(R, \gamma^{s}\right) .
$$

It is not difficult to see that $\operatorname{ker}(\beta)=D_{n}\left(\gamma^{e-1} R\right)$ and $|\operatorname{ker}(\beta)|=q^{n}$. Hence,

$$
\begin{aligned}
\mid\left\{A \in D_{n}(R) \mid\right. & \left.\operatorname{det}(\beta(A))=\gamma^{s}+\gamma^{e-1} R\right\} \mid \\
& =q^{n}\left|\left\{B \in D_{n}\left(R / \gamma^{e-1} R\right) \mid \operatorname{det}(B)=\gamma^{s}+\gamma^{e-1} R\right\}\right| \\
& =q^{n} d_{n}\left(R / \gamma^{e-1} R, \gamma^{s}+\gamma^{e-1} R\right) .
\end{aligned}
$$

From (3.3) and (3.4), it can be concluded that

$$
q d_{n}\left(R, \gamma^{s}\right)=q^{n} d_{n}\left(R / \gamma^{e-1} R, \gamma^{s}+\gamma^{e-1} R\right)
$$

As desired, we have

$$
d_{n}\left(R, \gamma^{s}\right)=q^{(n-1)} d_{n}\left(R / \gamma^{e-1} R, \gamma^{s}+\gamma^{e-1} R\right)
$$

The proof is completed.

Applying Lemma 3.7 recursively, the next corollary follows.

Corollary 3.8. Let $R$ be a CFCR of nilpotency index $e+f$ and residue field $\mathbb{F}_{q}$, where $2 \leq e$ and $1 \leq f$ are integers. Let $\gamma$ be a generator of the maximal ideal of $R$. Then

$$
d_{n}\left(R, \gamma^{s}\right)=q^{f(n-1)} d_{n}\left(R / \gamma^{e} R, \gamma^{s}+\gamma^{e} R\right)
$$

for all $1 \leq s<e$ and for all positive integers $n$.

The number $d_{n}\left(R, \gamma^{s}\right)$ is determined in the next theorem. 
Theorem 3.9. Let $R$ be a CFCR of nilpotency index e and residue field $\mathbb{F}_{q}$ and let $\gamma$ be a generator of the maximal ideal of $R$. Then

$$
d_{n}\left(R, \gamma^{s}\right)=q^{(e-1)(n-1)}(q-1)^{n-1}\left(\begin{array}{c}
n+s-1 \\
n-1
\end{array}\right)
$$

for all integers $1 \leq s<e$ and for all positive integers $n$.

Proof. Let $1 \leq s<e$ be an integer and let $n$ be a positive integer. Let $\mu: D_{n}\left(R / \gamma^{s+1} R\right) \rightarrow D_{n}\left(R / \gamma^{s} R\right)$ be a ring homomorphism defined by

$$
\mu(A)=\bar{A},
$$

where $\overline{\left[a_{i j}+\gamma^{s+1} R\right]}:=\left[a_{i j}+\gamma^{s} R\right]$ for all $\left[a_{i j}+\gamma^{s+1} R\right] \in D_{n}\left(R / \gamma^{s+1} R\right)$. For each $A \in D_{n}\left(R / \gamma^{s+1} R\right)$, we then have $\operatorname{det}(\mu(A))=0+\gamma^{s} R$ if and only if $\operatorname{det}(A)=\gamma^{s} b+\gamma^{s+1} R$ for some $b \in V$, where $V$ is given in Lemma 2.1. Since $|\operatorname{ker}(\mu)|=q^{n}$, it follows that

$$
\begin{aligned}
q^{n} d_{n} & \left(R / \gamma^{s} R, 0+\gamma^{s} R\right) \\
& =|\operatorname{ker}(\mu)| d_{n}\left(R / \gamma^{s} R, 0+\gamma^{s} R\right) \\
& =\sum_{b \in V} d_{n}\left(R / \gamma^{s+1} R, \gamma^{s} b+\gamma^{s+1} R\right) \\
& =d_{n}\left(R / \gamma^{s+1} R, 0+\gamma^{s+1} R\right)+\sum_{b \in V \backslash\{0\}} d_{n}\left(R / \gamma^{s+1} R, \gamma^{s} b+\gamma^{s+1} R\right) \\
& =d_{n}\left(R / \gamma^{s+1} R, 0+\gamma^{s+1} R\right)+(q-1) d_{n}\left(R / \gamma^{s+1} R, \gamma^{s}+\gamma^{s+1} R\right)
\end{aligned}
$$

by Theorem 3.1. Consequently,

$$
d_{n}\left(R / \gamma^{s+1} R, \gamma^{s}+\gamma^{s+1} R\right)=\frac{1}{q-1}\left(q^{n} d_{n}\left(R / \gamma^{s} R, 0+\gamma^{s} R\right)-d_{n}\left(R / \gamma^{s+1} R, 0+\gamma^{s+1} R\right)\right) .
$$

By Corollary 3.8 and the fact that $(s+1)+(e-s-1)=e$, we have

$$
d_{n}\left(R, \gamma^{s}\right)=q^{(e-s-1)(n-1)} d_{n}\left(R / \gamma^{s+1} R, \gamma^{s}+\gamma^{s+1} R\right) .
$$

From (3.5) and (3.6), we have

$$
d_{n}\left(R, \gamma^{s}\right)=\frac{q^{(e-s-1)(n-1)}}{q-1}\left(q^{n} d_{n}\left(R / \gamma^{s} R, 0+\gamma^{s} R\right)-d_{n}\left(R / \gamma^{s+1} R, 0+\gamma^{s+1} R\right)\right) .
$$

Using Theorem 3.6, it follows that

$$
\begin{aligned}
d_{n}\left(R, \gamma^{s}\right)= & \frac{q^{(e-s-1)(n-1)}}{q-1}\left(q^{n}\left(q^{n s}-(q-1)^{n} q^{(s-1) n} \sum_{i=0}^{s-1}\left(\begin{array}{c}
n+i-1 \\
n-1
\end{array}\right) q^{-i}\right)\right. \\
& \left.-\left(q^{n(s+1)}-(q-1)^{n} q^{s n} \sum_{i=0}^{s}\left(\begin{array}{c}
n+i-1 \\
n-1
\end{array}\right) q^{-i}\right)\right) \\
= & \frac{q^{(e-s-1)(n-1)}}{q-1}\left(q^{n(s+1)}-(q-1)^{n} q^{s n} \sum_{i=0}^{s-1}\left(\begin{array}{c}
n+i-1 \\
n-1
\end{array}\right) q^{-i}\right. \\
& \left.-q^{n(s+1)}+(q-1)^{n} q^{s n} \sum_{i=0}^{s}\left(\begin{array}{c}
n+i-1 \\
n-1
\end{array}\right) q^{-i}\right)
\end{aligned}
$$




$$
\begin{aligned}
= & \frac{q^{(e-s-1)(n-1)}}{q-1}\left(( q - 1 ) ^ { n } q ^ { s n } \left(\sum_{i=0}^{s}\left(\begin{array}{c}
n+i-1 \\
n-1
\end{array}\right) q^{-i}\right.\right. \\
& \left.\left.-\sum_{i=0}^{s-1}\left(\begin{array}{c}
n+i-1 \\
n-1
\end{array}\right) q^{-i}\right)\right) \\
= & \frac{q^{(e-s-1)(n-1)}}{q-1}(q-1)^{n} q^{s n}\left(\begin{array}{c}
n+s-1 \\
n-1
\end{array}\right) q^{-s} \\
= & q^{(e-1)(n-1)}(q-1)^{n-1}\left(\begin{array}{c}
n+s-1 \\
n-1
\end{array}\right)
\end{aligned}
$$

as desired.

\section{Circulant Matrices over CFCRs}

Determinants of circulant matrices over a CFCR are focused on and the number $c_{n}(R, a)$ of $n \times n$ circulant matrices of determinant $a$ is studied.

We recalled that $R$ denotes a CFCR of nilpotency index $e$, residue field $\mathbb{F}_{q}$, the maximal ideal generated $\gamma$. By Lemma 2.1, an element $a \in R$ can be written in the form $a=\gamma^{s} b$ for some $0 \leq s \leq e$ and unit $b \in U(R)$. Precisely, $a$ is a unit if $s=0, a=\gamma^{s} b$ is a zero-divisor if $1 \leq s \leq e-1$, and $a=0$ if $s=e$.

The over view results of the study of $c_{n}(R, a)$ are summarized in Figure 2. The solid blocks are hold true for all integers $n$ such that $\operatorname{gcd}(n, q)=1$ and the dashed blocks hold true for every positive integer $n$ such that $n \mid(q-1)$. The details and their proofs are given right after.

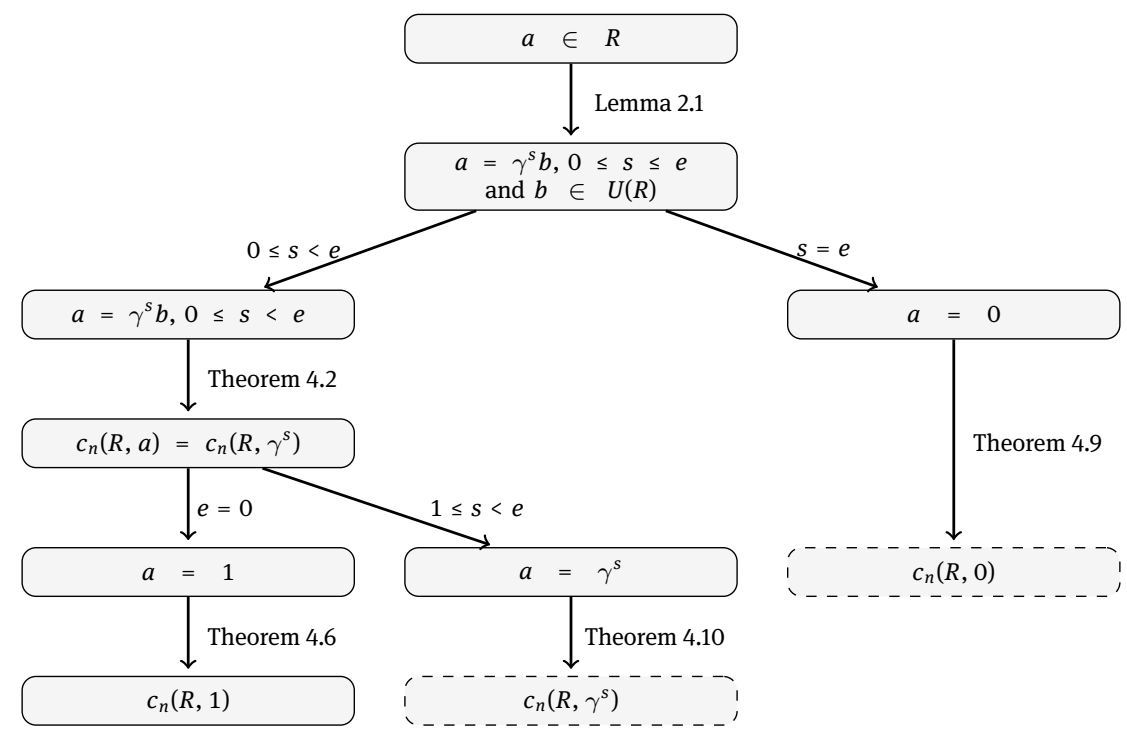

Figure 2: Steps in computing $c_{n}(R, a)$ over a CFCR $R$

A key relation in the study of $c_{n}(R, a)$ is given in Theorem 4.2. First, we prove a useful lemma.

Lemma 4.1. Let $R$ be a CFCR with residue field $\mathbb{F}_{q}$ and let $n$ be a positive integer. If $\operatorname{gcd}(n, q)=1$, then $\{\operatorname{det}(A) \mid$ $\left.A \in C_{n}(R)\right\}=R$. 
Proof. Assume that $\operatorname{gcd}(n, q)=1$. Let $Y=\{\operatorname{cir}(a, b, b, \ldots, b) \mid a, b \in R\}$. Using elementary row operations, it is not difficult to see that

$$
\operatorname{det}\left(\operatorname{cir}_{n}(a, b, b, \ldots, b)\right)=(a-b)^{n-1}(a+(n-1) b) .
$$

Let $c \in R$. Since $\operatorname{gcd}(n, q)=1, n$ is invertible in $R$. Choose $a=(c-(1-n)) n^{-1}$ and $b=(c-1) n^{-1}$ be elements in $R$. Then $a-b=(c-(1-n)) n^{-1}-(c-1) n^{-1}=1, a+(n-1) b=(c-(1-n)) n^{-1}+(n-1)(c-1) n^{-1}=c$, and hence,

$$
\operatorname{det}\left(\operatorname{cir}_{n}(a, b, b, \ldots, b)\right)=(a-b)^{n-1}(a+(n-1) b)=1^{n-1} c=c .
$$

Since $Y \subseteq C_{n}(R), R \subseteq\{\operatorname{det}(A) \mid A \in Y\} \subseteq\left\{\operatorname{det}(A) \mid A \in C_{n}(R)\right\} \subseteq R$. As desired, we have $\{\operatorname{det}(A) \mid A \in$ $\left.C_{n}(R)\right\}=R$.

Theorem 4.2. Let $R$ be a CFCR of nilpotency index e and residue field $\mathbb{F}_{q}$. Let $\gamma$ be a generator of the maximal ideal of $R$ and let $n$ be a positive integer. If $\operatorname{gcd}(n, q)=1$, then

$$
c_{n}\left(R, b \gamma^{s}\right)=c_{n}\left(R, \gamma^{s}\right)>0
$$

for all units $b$ in $U(R)$ and for all integers $0 \leq s \leq e$.

Proof. Let $0 \leq s \leq e$ be an integer and let $b$ be a unit in $U(R)$. In the case where $s=e$, we have $\gamma^{s}=0=\gamma^{s} b$. Hence, $c_{n}\left(R, \gamma^{s}\right)=c_{n}(R, 0)=c_{n}\left(R, b \gamma^{s}\right)$. By Lemma 4.1, we have $c_{n}\left(R, \gamma^{s}\right)>0$.

By Lemma 4.1, there exists a matrix $B \in C_{n}(R)$ such that $\operatorname{det}(B)=b$. For each $0 \leq s<e$, let $\alpha: C_{n}\left(R, \gamma^{s}\right) \rightarrow$ $C_{n}\left(R, b \gamma^{s}\right)$ be a map defined by

$$
\alpha(A)=B A
$$

for all $A \in C_{n}\left(R, \gamma^{S}\right)$. Since $B$ is nonsingular and $\operatorname{det}(A)=\gamma^{S}$ if and only if $\operatorname{det}(B A)=\operatorname{det}(B) \operatorname{det}(A)=b \gamma^{S}$ for all $A \in C_{n}\left(R, \gamma^{S}\right), \alpha$ is well-defined and bijective. Therefore, we have

$$
c_{n}\left(R, \gamma^{s}\right)=\left|C_{n}\left(R, \gamma^{s}\right)\right|=\left|C_{n}\left(R, b \gamma^{s}\right)\right|=c_{n}\left(R, b \gamma^{s}\right)
$$

as desired.

By setting $s=0$, the following corollary can be obtained immediately.

Corollary 4.3. Let $R$ be a CFCR with residue field $\mathbb{F}_{q}$ and let $n$ be a positive integer such that $\operatorname{gcd}(n, q)=1$. Then $c_{n}(R, a)=c_{n}(R, 1)$ for all $a \in U(R)$.

\subsection{Determinants of Nonsingular Circulant Matrices over CFCRs}

Nonsingular $n \times n$ circulant matrices over a CFCR are studied and the number $c_{n}(R, a)$ is determined for all $a \in U(R)$ and for all positive integers $n$ such that $\operatorname{gcd}(n, q)=1$. By Corollary 4.3, it is enough to derive only the number $c_{n}(R, 1)$.

Let $N_{S C}(R)=\left\{A \in C_{n}(R) \mid \operatorname{det}(A) \in U(R)\right\}$ denote the set of nonsingular $n \times n$ circulant matrices over a CFCR $R$. The number $\left|N S C_{n}\left(\mathbb{F}_{q}\right)\right|$ which is key to determine $c_{n}(R, 1)$ is given in [16, Proposition 1] and [7] via the ring isomorphism $T: C_{n}(R) \rightarrow R[X] /\left\langle X^{n}-1\right\rangle$ defined by

$$
\operatorname{cir}\left(a_{1}, a_{2}, \ldots, a_{n}\right) \mapsto a_{1}+a_{2} X+a_{3} X^{2}+\cdots+a_{n} X^{n-1}+\left\langle X^{n}-1\right\rangle .
$$

Lemma 4.4 ([16, Proposition 1] and [7]). Let $q$ be a prime power and let $n$ be a positive integer such that $\operatorname{gcd}(n, q)=1$. Then

$$
\left|N S C_{n}\left(\mathbb{F}_{q}\right)\right|=\prod_{d \mid n}\left(q^{\operatorname{ord}_{d}(q)}-1\right)^{\frac{\phi(d)}{\operatorname{ord}_{d}(q)}}=q^{n} \prod_{d \mid n}\left(1-q^{-\operatorname{ord}_{d}(q)}\right)^{\frac{\phi(d)}{\operatorname{ord}_{d}(q)}} .
$$


In general, we have

$$
\left|N S C_{n p^{k}}\left(\mathbb{F}_{q}\right)\right|=q^{n p^{k}} \prod_{d \mid n}\left(1-q^{-\operatorname{ord}_{d}(q)}\right)^{\frac{\phi(d)}{\operatorname{ord}_{d}(q)}}
$$

where $k \geq 0$ is a positive integer and $p$ is the characteristic of $\mathbb{F}_{q}$.

The cardinality of $\operatorname{NSC}_{n}(R)$ is given in the following lemma.

Lemma 4.5. Let $R$ be a CFCR of nilpotency index e and residue field $\mathbb{F}_{q}$ and let $n$ be a positive integer such that $\operatorname{gcd}(n, q)=1$. Then

$$
\left|N S C_{n}(R)\right|=q^{e n} \prod_{d \mid n}\left(1-q^{-\operatorname{ord}_{d}(q)}\right)^{\frac{\phi(d)}{\operatorname{ord}_{d}(q)}}
$$

Proof. For $e=1$, we have $R=\mathbb{F} q$ and

$$
\left|N S C_{n}(R)\right|=\left|N S C_{n}\left(\mathbb{F}_{q}\right)\right|=q^{n} \prod_{d \mid n}\left(1-q^{-\operatorname{ord}_{d}(q)}\right)^{\frac{\phi(d)}{\operatorname{ord}_{d}(q)}}
$$

by Lemma 4.4 .

Assume that $e \geq 2$. Let $\gamma$ be a generator of the maximal ideal of $R$ and let $\beta: C_{n}(R) \rightarrow C_{n}\left(R / \gamma^{e-1} R\right)$ be a ring homomorphism defined by

$$
\beta(A)=\bar{A}
$$

where $\overline{\left[a_{i j}\right]}:=\left[a_{i j}+\gamma^{e-1} R\right]$ for all $\left[a_{i j}\right] \in C_{n}(R)$. Then $A \in \operatorname{ker}(\beta)$ if and only if the entries of $A$ are in $\gamma^{e-1} R$. Or equivalently, $A \in C_{n}\left(\gamma^{e-1} R\right)$. By Lemma 2.1, $\left|\gamma^{e-1} R\right|=q$ which implies that $|\operatorname{ker}(\beta)|=\left|\gamma^{e-1} R\right|^{n}=q^{n}$. By the 1st Isomorphism Theorem, it follows that

$$
\left|C_{n}(R)\right|=|\operatorname{ker}(\beta)|\left|C_{n}\left(R / \gamma^{e-1} R\right)\right|=q^{n}\left|C_{n}\left(R / \gamma^{e-1} R\right)\right| .
$$

For each $B \in C_{n}\left(R / \gamma^{e-1} R\right)$, we have $\beta^{-1}(B)=\{A+\operatorname{ker}(\beta)\}$, where $A \in C_{n}(R)$ is such that $\beta(A)=B$. Note that $A \in C_{n}(R)$ is invertible if and only if $\beta(A)$ is a unit in $M_{n}\left(R / \gamma^{e-1} R\right)$. For each $B \in N S C_{n}\left(R / \gamma^{e-1} R\right)$, we therefore have

$$
\beta^{-1}(B) \subseteq N S C_{n}(R) \text { and }\left|\beta^{-1}(B)\right|=|\operatorname{ker}(\beta)|
$$

It follows that

$$
\left|N S C_{n}(R)\right|=|\operatorname{ker}(\beta)|\left|N S C_{n}\left(R / \gamma^{e-1} R\right)\right|=q^{n}\left|N S C_{n}\left(R / \gamma^{e-1} R\right)\right|
$$

Continue this process, it can be concluded that

$$
\begin{aligned}
\left|N S C_{n}(R)\right| & =q^{n}\left|N S C_{n}\left(R / \gamma^{e-1} R\right)\right| \\
& =q^{n} q^{n}\left|N S C_{n}\left(R / \gamma^{e-2} R\right)\right| \\
& \vdots \\
& =q^{(e-1) n}\left|N S C_{n}(R / \gamma R)\right| \\
& =q^{(e-1) n}\left|N S C_{n}\left(\mathbb{F}_{q}\right)\right| .
\end{aligned}
$$

Since $\left|N S C_{n}\left(\mathbb{F}_{q}\right)\right|=q^{n} \prod_{d \mid n}\left(1-q^{-\operatorname{ord}_{d}(q)}\right)^{\frac{\phi(d)}{\operatorname{ord}_{d}^{(q)}}}$ by Lemma 4.4, it follows that

$$
\left|N S C_{n}(R)\right|=q^{(e-1) n}\left|N S C_{n}\left(\mathbb{F}_{q}\right)\right|=q^{e n} \prod_{d \mid n}\left(1-q^{-\operatorname{ord}_{d}(q)}\right)^{\frac{\phi(d)}{\operatorname{ord}_{d}(q)}}
$$

as desired. 
The number $c_{n}(R, 1)$ is given in the next theorem for all positive integers such that $\operatorname{gcd}(n, q)=1$.

Theorem 4.6. Let $R$ be a CFCR of nilpotency index $e$ and residue field $\mathbb{F}_{q}$ and let $n$ be a positive integer such that $\operatorname{gcd}(n, q)=1$. Then

$$
c_{n}(R, 1)=q^{e(n-1)} \prod_{d \mid n, d \neq 1}\left(1-q^{-\operatorname{ord}_{d}(q)}\right)^{\frac{\phi(d)}{\operatorname{ord}_{d}(q)}} .
$$

Proof. Clearly, $\operatorname{NSC}_{n}(R)$ is the disjoint union of $C_{n}(R, a)$ for all $a \in U(R)$, i.e.,

$$
N S C_{n}(R)=\bigcup_{a \in U(R)} C_{n}(R, a)
$$

and $C_{n}(R, a) \cap C_{n}(R, b)=\emptyset$ for all $a \neq b$ in $U(R)$.

From Corollary 4.3, we have $c_{n}(R, 1)=c_{n}(R, a)=\left|C_{n}(R, a)\right|$ for all units $a \in U(R)$. It follows that

$$
\left|N S C_{n}(R)\right|=\sum_{a \in U(R)}\left|C_{n}(R, a)\right|=|U(R)| C_{n}(R, 1) .
$$

By Lemma 2.1 and Lemma 4.5, we therefore have

$$
\begin{aligned}
c_{n}(R, 1) & =\frac{\left|N S C_{n}(R)\right|}{|U(R)|} \\
& =\frac{q^{e n} \prod_{d \mid n}\left(1-q^{-\operatorname{ord}_{d}(q)}\right)^{\frac{\phi(d)}{\operatorname{ord}_{d}(q)}}}{(q-1) q^{e-1}} \\
& =q^{e(n-1)} \prod_{d \mid n, d \neq 1}\left(1-q^{-\operatorname{ord}_{d}(q)}\right)^{\frac{\phi(d)}{\text { ord }_{d}(q)}}
\end{aligned}
$$

as desired.

From Corollary 4.3 and Theorem 4.6, the next corollary can be deduced directly.

Corollary 4.7. Let $R$ be a CFCR of nilpotency index $e$ and residue field $\mathbb{F}_{q}$ and let $n$ be a positive integer such that $\operatorname{gcd}(n, q)=1$. Then

$$
c_{n}(R, a)=c_{n}(R, 1)=q^{e(n-1)} \prod_{d \mid n, d \neq 1}\left(1-q^{-\operatorname{ord}_{d}(q)}\right)^{\frac{\phi(d)}{\operatorname{ord}_{d}(q)}}
$$

for all units $a$ in $R$.

\subsection{Determinants of Singular Circulant Matrices over CFCRs}

We focus on singular $n \times n$ circulant matrices over a CFCR $R$ with residue field $\mathbb{F}_{q}$ and determine the number $c_{n}(R, a)$ of such matrices for all $a \in \gamma R$ in the special case where $n$ is a positive divisor of $q-1$.

First, a key relation between $c_{n}(R, a)$ and $d_{n}(R, a)$ is given for all $a \in R$ and for all positive divisors $n$ of $q-1$.

Lemma 4.8. Let $R$ be a CFCR of with residue field $\mathbb{F}_{q}$ and let $n$ be a positive integer. If $n \mid(q-1)$, then $c_{n}(R, a)=$ $d_{n}(R, a)$ for all $a \in R$. 
Proof. Assume that $n$ is a divisor of $q-1=|V \backslash\{0\}|$. Then $R$ contains an $n$th root of unity in $V$ and denote it by $\omega$. Let $P=\left[p_{i j}\right]$ be the $n \times n$ matrix over $R$ defined by $p_{i j}=\omega^{(i-1)(j-1)}$ for all $1 \leq i \leq n$ and $1 \leq j \leq n$. Precisely,

$$
P=\left[\begin{array}{ccccc}
1 & 1 & 1 & \ldots & 1 \\
1 & \omega^{1(1)} & \omega^{1(2)} & \ldots & \omega^{1(n-1)} \\
1 & \omega^{2(1)} & \omega^{2(2)} & \ldots & \omega^{2(n-1)} \\
\vdots & \vdots & \vdots & \ddots & \vdots \\
1 & \omega^{(n-1)(1)} & \omega^{(n-1)(2)} & \ldots & \omega^{(n-1)(n-1)}
\end{array}\right] .
$$

Using the arguments similar to those in [4, Page 74] and the fact that $\operatorname{gcd}(n, q)=1, P$ is invertible. Let $A=$ $\operatorname{cir}\left(a_{1}, a_{2}, \ldots, a_{n}\right) \in C_{n}(R)$. From (2.1), the eigenvalues of $A$ are of the form

$$
\omega_{j}=\sum_{i=1}^{n} a_{i} \omega^{(i-1) j}
$$

for all $0 \leq j \leq n-1$. By the direct calculation (see [4] and [14]), it follows that $A$ is diagonalizable with $P A P^{-1}=D$ and $D=\operatorname{diag}\left(\omega_{0}, \omega_{1}, \ldots, \omega_{n-1}\right)$.

Let $\theta: C_{n}(R) \rightarrow D_{n}(R)$ be the determinant preserving ring homomorphism defined by

$$
A \mapsto P A P^{-1}=D
$$

for all $A \in C_{n}(R)$. Let $B \in \operatorname{ker}(\theta)$. Then $[0]=\theta(B)=P B P^{-1}$. Since $P$ is invertible, $B=P^{-1}[0] P=[0]$. Then $\operatorname{ker}(\theta)=\{[0]\}$ which implies that $\theta$ is injective. Since $\left|C_{n}(R)\right|=|R|^{n}=\left|D_{n}(R)\right|$, it follows that $\theta$ is a bijection. Hence, it is a determinant preserving ring isomorphism. Consequently, $c_{n}(R, a)=d_{n}(R, a)$ for all $a \in R$.

From Lemma 4.8, we have that $c_{n}(R, 0)=d_{n}(R, 0)$ and $d_{n}(R, 0)$ is determined in Theorem 3.6. Hence, the next theorem follows.

Theorem 4.9. Let $R$ be a CFCR of nilpotency index e and residue field $\mathbb{F}_{q}$ and let $n$ be a positive divisor of $q-1$. Then

$$
c_{n}(R, 0)=q^{n e}-(q-1)^{n} q^{(e-1) n} \sum_{i=0}^{e-1}\left(\begin{array}{c}
n+i-1 \\
n-1
\end{array}\right) q^{-i} .
$$

From Theorem 4.2, we have $c_{n}\left(R, \gamma^{s} b\right)=c_{n}\left(R, \gamma^{s}\right)$. By Lemma 4.8, it follows that $c_{n}\left(R, \gamma^{s}\right)=d_{n}\left(R, \gamma^{s}\right)$ which is determined in Theorem 3.9. The next theorem therefore follows.

Theorem 4.10. Let $R$ be a CFCR of nilpotency index e and residue field $\mathbb{F}_{q}$ and let $n$ be a positive divisor of $q-1$. Let $\gamma$ be a generator of the maximal ideal of $R$ and let $b \in U(R)$. Then

$$
c_{n}\left(R, \gamma^{s} b\right)=c_{n}\left(R, \gamma^{s}\right)=q^{(e-1)(n-1)}(q-1)^{n-1}\left(\begin{array}{c}
n+s-1 \\
n-1
\end{array}\right)
$$

for all integers $1 \leq s<e$.

\section{Conclusion and Remarks}

Summary, conjectures, open problems are given in this section together with a brief discussion on the determinants of diagonal and circulant matrices over commutative finite principal ideal rings (CFPIRs).

\subsection{Conclusion}

Determinants of $n \times n$ matrices over CFCRs $R$ with residue field $\mathbb{F}_{q}$ have been established in [5]. Here, determinants of $n \times n$ diagonal and circulant matrices over $R$ which are subrings of the matrices in [5] have been 
studied. The number of $n \times n$ diagonal matrices over $R$ of a fixed determinant $a$ has been completely determined for all positive integers $n$ and for all elements $a \in R$. For nonsingular circulant matrices, the number of $n \times n$ circulant matrices over $R$ whose determinant is a unit in $R$ has been completely determined for all units in $R$ and for all positive integers $n$ such that $\operatorname{gcd}(n, q)=1$. For singular circulant matrices, the number of $n \times n$ circulant matrices over $R$ whose determinants are non-units has been completely determined for all non-units in $R$ and positive divisors $n$ of $q-1$. The other cases remain as open problems.

\subsection{Determinants of Diagonal and Circulant Matrices over CFPIRs}

A ring $\mathcal{R}$ with identity $1 \neq 0$ is called a commutative finite principal ideal ring (CFPIR) if $\mathcal{R}$ is finite, commutative, and every ideal in $\mathcal{R}$ is principal. It is well known (see [8]) that every CFPIR is a direct product of CFCRs. Hence, a CFPIR $\mathcal{R}$ can be written in the form of $\mathcal{R}=R_{1} \times R_{2} \times \cdots \times R_{m}$ for some positive integer $m$, where $R_{i}$ is a CFCR for all $1 \leq i \leq m$.

For each $1 \leq i \leq m$, let $\phi_{i}: \mathcal{R} \rightarrow R_{i}$ be a surjective ring homomorphism defined by

$$
\phi_{i}\left(\left(r_{1}, r_{2}, \ldots, r_{m}\right)\right)=r_{i} .
$$

Let $M_{n}(\mathcal{R})$ be the ring of $n \times n$ matrices over $\mathcal{R}$. In [5, Theorem 4.1], it has been shown that the map $\Phi: M_{n}(\mathcal{R}) \rightarrow$ $M_{n}\left(R_{1}\right) \times M_{n}\left(R_{2}\right) \times \cdots \times M_{n}\left(R_{m}\right)$ defined by

$$
\left[a_{i j}\right] \mapsto\left(\left[\phi_{1}\left(a_{i j}\right)\right],\left[\phi_{2}\left(a_{i j}\right)\right], \ldots,\left[\phi_{m}\left(a_{i j}\right)\right]\right)
$$

is a ring isomorphism. Since $D_{n}(\mathcal{R})$ and $C_{n}(\mathcal{R})$ are subrings of $M_{n}(\mathcal{R})$, the restriction maps $\left.\Phi\right|_{D_{n}(\mathcal{R})}: D_{n}(\mathcal{R}) \rightarrow$ $D_{n}\left(R_{1}\right) \times D_{n}\left(R_{2}\right) \times \cdots \times D_{n}\left(R_{m}\right)$ and $\left.\Phi\right|_{C_{n}(\mathcal{R})}: C_{n}(\mathcal{R}) \rightarrow C_{n}\left(R_{1}\right) \times C_{n}\left(R_{2}\right) \times \cdots \times C_{n}\left(R_{m}\right)$ are ring isomorphisms. Consequently, the following theorem can be obtained using the arguments similar to those in the proof of [5, Theorem 4.1].

Theorem 5.1. Let $\mathcal{R}=R_{1} \times R_{2} \times \cdots \times R_{m}$ be a CFPIR where $R_{1}, R_{2}, \ldots, R_{m}$ be CFCRs and let $n$ be a positive integer. Let $r \in \mathcal{R}$ and let $\phi_{i}$ 's be defined as above. Then

$$
d_{n}(\mathcal{R}, r)=d_{n}\left(R_{1}, \phi_{1}(r)\right) d_{n}\left(R_{2}, \phi_{2}(r)\right) \ldots d_{n}\left(R_{m}, \phi_{m}(r)\right)
$$

and

$$
c_{n}(\mathcal{R}, r)=c_{n}\left(R_{1}, \phi_{1}(r)\right) c_{n}\left(R_{2}, \phi_{2}(r)\right) \ldots c_{n}\left(R_{m}, \phi_{m}(r)\right)
$$

\subsection{Conjectures and Open Problems}

Based on our observation, we conjecture that the condition $\operatorname{gcd}(n, q)=1$ in Theorem 4.2 can be omitted. However, the original proof of Theorem 4.2 does not work.

Conjecture 5.2. Let $R$ be a CFCR of nilpotency index e and let $n$ be a positive integer. If the maximal ideal of $R$ is generated by $\gamma$, then

$$
c_{n}\left(R, \gamma^{s}\right)=c_{n}\left(R, b \gamma^{s}\right)
$$

for all units $b$ in $U(R)$ and $0 \leq s \leq e$. This number can be zero.

In Lemma 4.1, $\left\{\operatorname{det}(A) \mid A \in C_{n}(R)\right\}=R$ for all positive integers $n$ such that $\operatorname{gcd}(n, q)=1$. Once $\operatorname{gcd}(n, q) \neq 1$, $\left\{\operatorname{det}(A) \mid A \in C_{n}(R)\right\}$ does not need to equal $R$, e.g., there are no $2 \times 2$ matrices over $\mathbb{Z}_{4}$ whose determinant is a zero-divisor 2 . We have the following conjecture.

Conjecture 5.3. Let $R$ be a CFCR of nilpotency index e and let $n$ be a positive integer. Then $U(R) \subseteq\{\operatorname{det}(A) \mid$ $\left.A \in C_{n}(R)\right\}$. Precisely, $C_{n}(R, a)>0$ for all units $a \in U(R)$. 
In general, it is interesting to solve the following open problems.

Problem 5.4. For a CFCR $R$ with residue field $\mathbb{F}_{q}$, determine $c_{n}(R, a)$ for all $a \in U(R)$ and for all positive integers $n$ such $\operatorname{gcd}(n, q) \neq 1$.

Problem 5.5. For a CFCR $R$ with residue field $\mathbb{F}_{q}$, determine $c_{n}(R, a)$ for all non-units $a \in \gamma R$ and for all positive integers $n$ which are non-divisors of $q-1$.

Problem 5.6. For a commutative finite ring $\mathfrak{R}$ and $a \in \mathfrak{R}$, determine $d_{n}(\mathfrak{R}, a)$ and $c_{n}(\mathfrak{R}, a)$ for all positive integers $n$.

Acknowledgements: This research was supported by the Thailand Research Fund and Silpakorn University under Research Grant RSA6280042. The authors would like to thank anonymous referees for useful comments.

\section{References}

[1] Alahmadi, A., Özdemir, F., Solé, P.: On self-dual double circulant codes, Designs, Codes and Cryptography 86, 1257-1265 (2018).

[2] Cao, Y.: Association schemes and directed graphs determined by orbitals of general linear groups over finite chain rings, Communication in Algebra 39, 220-236 (2011).

[3] Catalan, E.: Récherches sur les Déterminants, Bulletin de l’Academie Royale de Belgique 13, 534-555 (1846).

[4] Chalkley, R.: Circulant matrices and algebraic equations, Mathematics Magazine 48, 73-80 (1975).

[5] Choosuwan, P., Jitman, S., Udomkavanich, P.: Determinants of matrices over commutative finite principal ideal rings, Finite Fields and Their Applications 48, 126-140 (2017).

[6] Davis, P. J.: Circulant Matrices, 2nd edition, New York, Chelesa publishing, 1994.

[7] Fabšič, T. Grošek, O., Nemoga, K., Zajac, P.: On generating invertible circulant binary matrices with a prescribed number of ones, Cryptography and Communications 10, 159-175 (2018).

[8] Gilberto, B., Flaminio, F.: Finite Commutative Rings and Their Applications, Springer, 2002.

[9] Grassl, M., Gulliver, T. A.: On circulant self-dual codes over small fields, Designs, Codes and Cryptography 52, 57-81 (2009) -

[10] Gulliver, T. A., Harada, M.: Double circulant self-dual codes over $\mathbb{Z}_{2^{k}}$, IEEE Transactions on Information Theory 44, 3105-3123 (1998).

[11] Han, S., Kim, J.-L.: Computational results of duadic double circulant codes, Journal of Applied Mathematics and Computing 40, 33-43 (2012).

[12] Hou, X.: Finite commutative chain rings, Finite Fields and Their Applications 7, 382-396 (2001).

[13] Hou, X., Leung, K. H., Ma, S. L.: On the groups of units of finite commutative chain rings, Finite Fields and Their Applications 9, 20-38 (2003).

[14] Kra, I., Simanca, S. R.: On circulant matrices, Notices of the AMS 59, 368-377 (2012).

[15] Mukhopadhyay, A.: On the probability that the determinant of an $n \times n$ matrix over a finite field vanishes, Discrete Mathematics 51, 311-315 (1984).

[16] Samardjiska, S., Santini, P., Persichetti, E., Banegas, G.: A reaction attack against cryptosystems based on LRPC codes, Lecture Notes in Computer Science 11774, Springer, Cham, 2019.

[17] Shi, M., Huang, D., Sok, L., Solé, P.: Double circulant LCD codes over $\mathbb{Z}_{4}$, Finite Fields and Their Applications 58, 133-144 (2019).

[18] Shi, M., Zhu, H., Qian, L., Solé, P.: On self-dual four circulant codes, International Journal of Foundations of Computer Science 29, 1143-1150 (2018).

[19] van Asch, B. Matrix-product codes over finite chain rings, Applicable Algebra in Engineering, Communication and Computing 19, 39-49 (2008). 